\title{
THE EFFECTS OF FILTERS AND COLOUR ON STELLAR \\ OCCULTATIONS AND APPROPRIATE DECONVOLUTION PROCEDURES
}

\author{
T. KRISHNAN \\ Astro Research Corporation, P. O. Box 4128, Santa Barbara, Calif. 93103, U.S.A.
}

\begin{abstract}
The theory of the effect of bandwidth of lunar occultations is reviewed. It is recalled that effective beamshapes can be calculated for symmetrical bandpasses and that their widths are related to the absolute width in wavelength of the bandpasses. Restoration with the second differential of the theoretical Fresnel diffraction curves at the central wavelength, at the correct rates, yield source distributions as viewed by these beamshapes. It is shown that for asymmetric bandpasses, the real and odd parts taken about the centroids lead to equivalent even and odd beams. Assuming an approximate color temperature for the stars, the total system response can be evaluated and hence the even and odd parts. Restoration of the data should then be performed using the second differential of the Fresnel curve at the centroid wavelength to minimize the odd part, adjusting zeroes, rates, and centroids by inspection. The even part should then represent the even theoretical response convolved with the one-dimensional stellar distribution, provided the latter is circularly symmetrical.

The technique is applied to the occultation observation of $\lambda$-Aquarii by Nather et al. (1970) leading to closely similar results.
\end{abstract}

\section{Introduction}

It is obviously unnecessary to dwell at length to an audience such as this on the importance of measuring stellar diameters in astronomy. Suffice it to say that it is of importance to the study of stellar evolution and of stellar interiors.

The use of the method of lunar occultations to determine stellar diameters has been considered off and on for very many years, but it can be clearly divided into the preradio astronomy and post-radio astronomy phases. Early suggestions and observations include those of McMahon (1908), Eddington (1909), Williams (1939), and Whitford (1939) and show a progression from Eddington's early skepticism to Williams' optimism on the theoretical side. Observations by optical astronomers, notably those of Cousins and Guelke (1953), Evans et al. (1953), and Evans (1951, 1955, 1957), were made of improvingly higher quality. All analytical methods used by the astronomers then, and sometimes even now, involve fitting the data to model occultations.

The post-radio astronomy phase was marked by a publication by Scheuer in 1962, describing the theoretical basis for the conclusion that a Fresnel diffraction curve contained all the information that could be obtained if geometrical optics held. He was also able to show how the one-dimensional brightness distribution of a radio source could be 'restored'. Von Hoerner (1964) extended Scheuer's purely monochromatic theory to the consideration in radio astronomy of numerous criteria, such as signal-to-noise ratio, bandwidth, etc., on a semi-empirical basis. Since then Taylor (1966) and Berg (1969) have applied a 'Scheuer-Von Hoerner' method to optical occultations of stars but essentially use the monochromatic theory. 
The effect of finite bandpasses which are symmetric in wavelength have been explored by Scheuer (1965), Sutton (1966), Cohen (1969) and Krishnan (1970), all of whom arrived at the conclusion that the effective resolution due to smearing is proportional in such cases to the square root of the absolute bandwidth (or some measure of it, such as its half-width). Lang (1969) has also considered the effect of bandwidth adopting a slightly more complex approach of local co-sinusoidal approximations.

It has been customary in restoring occultation observations by the 'Scheuer-Von Hoerner' method to convolve the restoring function initially with a well-behaved curve such as a gaussian before convolving it with the data. In my paper of 1970 , I showed that this initial convolution was unnecessary in the presence, as is always the case, of a finite bandpass and that if the bandpass function were known, the effective beam could be calculated. In this paper I shall briefly outline the theory as put forward in that paper and extend it to the consideration of asymmetrical bandpasses as encountered in optical astronomy. The theory is simple in form, but the application is difficult to describe. I shall therefore discuss the application in the context of the observation in 1969 by Nather et al. (1970) of the occultation of $\lambda$-Aquarii, the raw data of which was kindly provided by the authors, and compare the conclusion with those arrived at by them using model fitting.

\section{Monochromatic Theory}

We begin by summarizing the monochromatic theory due to Scheuer (1962), expressing his equations in terms of $\theta$, the angular distance, in radians, of the source from

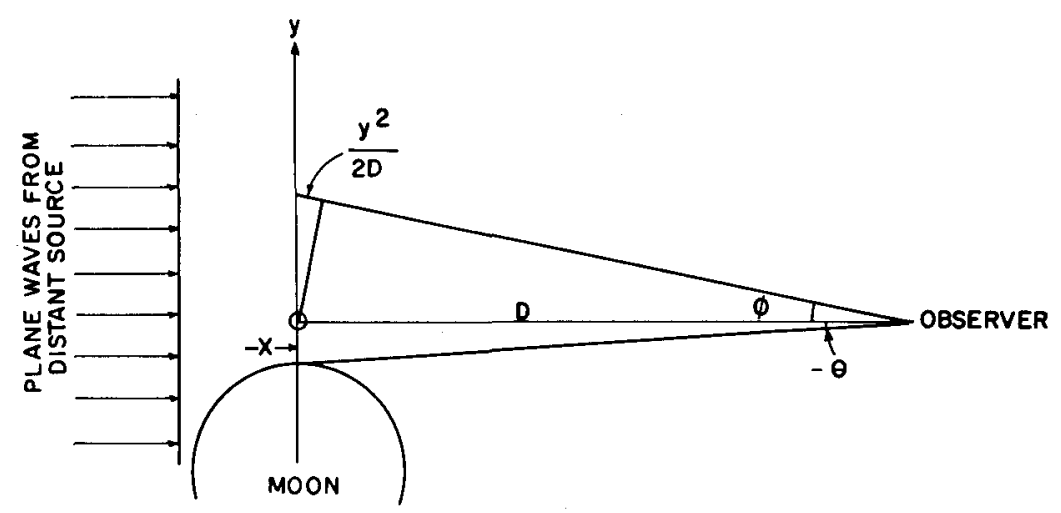

Fig. 1. Geometry of a lunar occultation of a star.

the edge of the Moon (Figure 1). $D$ is the distance to the Moon from the observer, and $\lambda$ is the wavelength of the radiation.

Provided the resolution sought is $\gg \lambda / D\left(\sim 10^{-10 "}\right.$ in the optical case), which is safe in all practical cases and particularly where the limit is set by the bandpass, the 
amplitude response due to an unit point source is given by

where

$$
A(\theta, \lambda)=\sqrt{b} \int_{-\theta}^{\infty} \exp \left(-i \pi b \Phi^{2}\right) \mathrm{d} \Phi
$$

$$
b=D / \lambda .
$$

The intensity pattern is given by

$$
p(\theta, \lambda)=b\left[\int_{-\theta}^{\infty} \exp \left(-i \pi b \Phi^{2}\right) \mathrm{d} \Phi \int_{-\theta}^{\infty} \exp \left(+i \pi b \Phi^{2}\right) \mathrm{d} \Phi\right] .
$$

For a source of finite size, but small enough for the Moon to still be considered a straight edge, the observed intensity is given by

$$
f(\theta, \lambda)=p(\theta, \lambda) * t(\theta, \lambda) \quad[* \text { denotes convolution }]
$$

where $t(\theta, \lambda)$ is the one-dimensional or strip distribution across the source measured in the direction perpendicular to the Moon's edge at the point of occultation.

Scheuer (1962), from consideration of the differential of the theoretical response and its Fourier Transform, shows that it is possible to restore the true distribution $t(\theta, \lambda)$ by convolution with a restoring function. If $q(\theta, \lambda)$ and $p^{\prime \prime}(\theta, \lambda)$ denote the first and second differentials of $p(\theta, \lambda)$, and $g(\theta, \lambda)$ the first differential of $f(\theta, \lambda)$, the important expressions in our notation become

$$
Q(s, \lambda)=\int_{-\infty}^{\infty} q(\theta, \lambda) \exp (-i \theta s) \mathrm{d} \theta=\exp \left(\frac{-i s^{2}}{4 \pi b} \operatorname{sgn} s\right)
$$

and

$$
t(\theta, \lambda)=q(-\theta, \lambda) * g(\theta, \lambda)
$$

Equation (1) shows that, in theory, all the Fourier components of the source are present with equal weight, but different phases. However, this is strictly not true as the Fourier Transform exists only when the pattern is truncated by observation after a finite period of time (normally by noise) as pointed out by Cohen (1969).

Scheuer (1962) is able to show that (2) is equivalent to the statement

$$
t(\theta, \lambda)=-p^{\prime \prime}(-\theta, \lambda) * f(\theta, \lambda)
$$

thus avoiding differentiation.

The restoring function $-p^{\prime \prime}(-\theta, \lambda)$ is 'badly behaved' and Scheuer suggested that it be made 'well behaved' by convolution with a 'well-behaved' function such as gaussian. The well-behaved function then defines the 'effective' beam of the observation.

* In my paper (Krishnan, 1970) dealing with narrow symmetrical bandpasses, $b$ occurs in this expression wrongly as an amplitude factor. 
$-p^{\prime \prime}(-\theta, \lambda)$ is obtained from expansions of the Fresnel series for $C(v)$ and $S(v)$ (see Von Hoerner, 1964) where $v$ is related to $\theta$ and $\lambda$ by the expression

$$
v=\theta \sqrt{\frac{2 D}{\lambda}} .
$$

\section{Wideband Theory}

In my paper (Krishnan, 1970) I have worked out the general theory of the effect of finite receiving bandpasses on the occultation pattern when the radiation from the source is spectrally flat. The error that I made in including an amplitude factor of $b$ (containing $\lambda$ ) in the expression for $Q(s, \lambda)$, noted in the previous section, led me to qualify my theory as being applicable only to narrow bandpasses. Since this factor drops out, the conclusions of that paper are applicable to wide bandpasses as well, and we shall briefly summarize the results.

At a given wavelength, $\lambda$, let the response of the receiving system be expressed as $m\left(\lambda-\lambda_{0}\right)$ or $m(l)$ where $l=\left(\lambda-\lambda_{0}\right)$. Then, by the monochromatic theory, if $f(\theta, \lambda)$ be the observed intensity and $g(\theta, \lambda)=\mathrm{d} / \mathrm{d} \theta[f(\theta, \lambda)]$,

$$
g(\theta, \lambda)=m\left(\lambda-\lambda_{0}\right)[t(\theta, \lambda) * q(\theta, \lambda)] .
$$

Let the observed intensity over the whole band be $f(\theta)$. We shall, for the purposes of theory, consider its first differential, $\dot{g}(\theta)$.

Then from the previous section

$$
\dot{g}(\theta)=\int_{\text {passband }} g(\theta, \lambda) \mathrm{d} \lambda=\int_{\text {passband }} m\left(\lambda-\lambda_{0}\right)[(t(\theta, \lambda) * q(\theta, \lambda] \mathrm{d} \lambda .
$$

If the radiation from the source is assumed to be spectrally flat over the passband as in most radio observations, we may denote the strip brightness distribution by that at a representative wavelength, $\lambda_{0}$, calling it $t\left(\theta, \lambda_{0}\right)$. $\lambda_{0}$, for a symmetrical bandpass is chosen to be the central wavelength.

$$
\begin{aligned}
\dot{g}(\theta) & =\int_{\text {passband }} m\left(\lambda-\lambda_{0}\right)\left[t\left(\theta, \lambda_{0}\right) * q(\theta, \lambda)\right] \mathrm{d} \lambda \\
& =t\left(\theta, \lambda_{0}\right) * \int_{\text {passband }} m\left(\lambda-\lambda_{0}\right) q(\theta, \lambda) \mathrm{d} \lambda .
\end{aligned}
$$

Let $\dot{G}(s), T\left(s, \lambda_{0}\right)$ and $R(s)$ represent the Fourier Transforms of $\dot{g}(\theta), t\left(\theta, \lambda_{0}\right)$ and $\int_{\text {passband }} m\left(\lambda-\lambda_{0}\right) q(\theta, \lambda) \mathrm{d} \lambda$ respectively. We adopt the convention for Fourier Transforms that are described by the transform pair

$$
F(s)=\int_{-\infty}^{\infty} f(x) \exp (-i x s) \mathrm{d} x
$$


and

Now,

$$
f(x)=\frac{1}{2 \pi} \int_{-\infty}^{\infty} F(s) \exp (i x s) \mathrm{d} x
$$

$$
\begin{aligned}
R(s) & =\int_{\text {passband }} m\left(\lambda-\lambda_{0}\right) Q(s, \lambda) \mathrm{d} \lambda \\
& =\int_{\text {passband }} m\left(\lambda-\lambda_{0}\right) \exp \left(\frac{\left.-i s^{2} \frac{\lambda}{4 \pi} \frac{1}{D} \operatorname{sgn} s\right) \mathrm{d} \lambda . \quad \text { (from (1)) }}{}\right.
\end{aligned}
$$

If we take transforms, Equation (4) becomes

$$
\dot{G}(s)=T\left(s, \lambda_{0}\right) R(s) .
$$

If $r(\theta)$ be the inverse $F . T$. of $R(s)$, taking inverse transforms,

$$
\dot{g}(\theta)=t\left(\theta, \lambda_{0}\right) * q\left(\theta, \lambda_{0}\right) * r(\theta)
$$

By analogy with Scheuer's derivation of Equation (3) it becomes possible to say

$$
t\left(\theta, \lambda_{0}\right) * r(\theta)=-p^{\prime \prime}\left(-\theta, \lambda_{0}\right) * f(\theta) * r(\theta)
$$

If $r(\theta)$ is 'well behaved' as it always will be if $m(l)$ involves a finite bounded filter, it represents an 'effective beam'. Under such conditions it is no longer necessary to

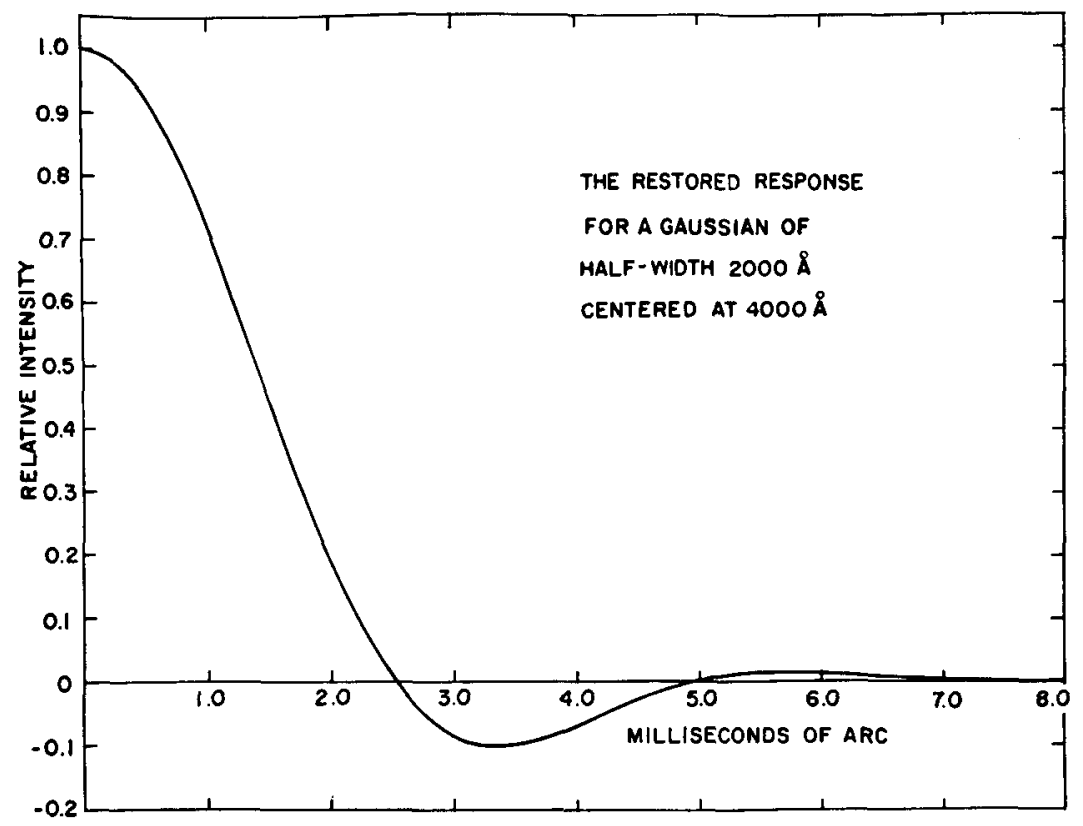

Fig. 2. The restored response for a symmetrical bandpass as obtained by computer simulation. 
preconvolve $-p^{\prime \prime}\left(-\theta, \lambda_{0}\right)$ with a well-behaved function. The passband performs this function automatically.

Restoration with $-p^{\prime \prime}\left(-\theta, \lambda_{0}\right)$ should be attempted directly provided an observational record of adequate length is available. The length of the restoring functon should extend to $-z$ units of $v$ if an effective beam width of $1.2 / z$ units of $v$ is to be obtained. When $m(l)$ is symmetrical about a central wavelength, $\lambda_{0}, r(\theta)$ is symmetrical about zero if the restoration is performed with $-p^{\prime \prime}\left(-\theta, \lambda_{0}\right)$.

With this theory behind us it is extremely simple to derive the 'effective beams' for various bandpasses which are symmetrical. In general, for various common bandpasses, the HPBW varies between 6.23 and 7.0 times $\sqrt{ } \Delta l$ arcsec for mean topocentric distance where $\Delta l$ is the half-power bandwidth. In my paper (Krishnan, 1970) it is also shown that among the bandpasses most commonly used the single-tuned bandpass of form $1 /\left(1+l^{2} / \sigma^{2}\right)$ affords the maximum sensitivity.

As an illustration of the validity of the theory for wide bandpasses, Figure 2 shows the restored response obtained by direct convolution of $-p^{\prime \prime}\left(-\theta, \lambda_{0}\right)\left(\lambda_{0}=4000 \AA\right)$ with the summed response from a point source, for a gaussian bandpass centered at $4000 \AA$ with a half-power bandwidth of $2000 \AA$. The HPBW is $0^{\prime \prime} .00286$, as calculated by theory. The calculations were carried out with the distance to the Moon as the mean topocentric distance of $3.794 \times 10^{10} \mathrm{~cm}$.

\section{Restoration of Optical Occultations}

Photoelectric photometry of lunar occultations adds two major problems to the application of the general theory. It also adds a host of minor problems associated with restoration, some of which involve one in iterative processes similar to those encountered in the reduction of radio occultations.

The two major problems are:

(a) asymmetry in the spectral response of the filter plus photometer and

(b) the spectral variation of stellar intensity with temperature, which is one of the unknowns.

The first is measurable, and if the temperature of the star is also known, the effective spectral response function, $\dot{m}(l)$, can be found. $\dot{m}(l)$ is in general asymmetric and hence $r(\theta)$ is also asymmetric, but to a lesser degree, since a transformation of variable from $s^{2}$ to $s$ takes place in going from $\dot{m}(l)$ to $r(\theta)$. Since our objective would be to determine stellar diameter and center-to-limb variation, if any, the important problem is how best to process the data. Major complications can be caused by the uncertainty in our knowledge of the lunar limb roughness at the point of occultation. Errors then arise in the zero and the rate of occultation, both of which are essential to a good restoration. In the case of observations with symmetric bandpasses, the error in phase of restoration can be detected by the loss of symmetry in the restored response, which will here be confused by the asymmetry of $r(\theta)$ itself. Further, any deduction about the stellar brightness distribution depends on going back from the strip distribution through models that assume circular symmetry. Any attempt at restoration implies 
careful consideration of the properties of symmetry that $r(\theta)$ might possess, and their utilization with minimal loss of information. It is just such a process that $I$ will now present and demonstrate its reasonable validity in application.

Let $E_{\lambda}$ be the intensity of the radiation from the star at wavelength $\lambda$, normally described by the Planck radiation law

$$
E_{\lambda} \mathrm{d} \lambda=\frac{h c^{2}}{\lambda^{5}} \frac{1}{e^{h c / k T \lambda}-1} \mathrm{~d} \lambda .
$$

We are now forced to assume, in order to progress with our theory, that the spectral behavior of the source does not change from strip to strip over the passband of the filter. Then, as the wavelength changes, the intensity in any strip changes in the same ratio as in the other strips. It also now becomes important to redefine $r(\theta)$ as $r\left(\theta, \lambda_{0}\right)$ where $\lambda_{0}$ is the wavelength used as reference. The spectrum of the whole source stays incorporated in the expressions, but $r\left(\theta, \lambda_{0}\right)$ will have different zeros for different choices of $\lambda_{0}$.

where

$$
t_{m}\left(\theta, \lambda_{0}\right) * r\left(\theta, \lambda_{0}\right)=\dot{f}(\theta) *-p^{\prime \prime}\left(-\theta, \lambda_{0}\right) * r\left(\theta, \lambda_{0}\right)
$$

$$
r\left(\theta, \lambda_{0}\right)=\frac{1}{2 \pi} \int_{-\infty}^{\infty} R\left(s, \lambda_{0}\right) \exp (-i \theta s) \mathrm{d} s
$$

and

$$
\begin{aligned}
R\left(s, \lambda_{0}\right) & =\int_{-\infty}^{\infty} \frac{E_{\lambda}}{E_{\lambda_{0}}} m(l) \exp \left(\frac{-i s^{2}}{4 \pi D} l \operatorname{sgn} s\right) \mathrm{d} l \\
& =\int_{-\infty}^{\infty} \dot{m}(l) \exp \left(\frac{-i s^{2}}{4 \pi D} l \operatorname{sgn} s\right) \mathrm{d} l
\end{aligned}
$$

where

$$
\dot{m}(l)=\frac{E_{\lambda}}{E_{\lambda_{0}}} m(l) .
$$

Thus the restoration can only provide a measure of the weighted mean value $t_{m}\left(\theta, \lambda_{0}\right)$, over the total spectral response, of the strip-integrated intensity distribution.

We begin by assuming that the temperature of the star is known and hence its spectral function. Further, we assume that the spectral response of the photoelectric system has been measured. Thus $\dot{m}(l)$ is fully known. We know that if we convolve the observational data correctly with $-p^{\prime \prime}\left(-\theta, \lambda_{0}\right)$ where $\lambda_{0}$ is the chosen reference frequency we shall obtain

$$
t_{m}\left(\theta, \lambda_{0}\right) * r\left(\theta, \lambda_{0}\right)
$$


Now,

$$
r\left(\theta, \lambda_{0}\right)=\frac{1}{2 \pi} \int_{-\infty}^{\infty} R\left(s, \lambda_{0}\right) \exp (i s \theta) \mathrm{d} s
$$

where

$$
R\left(s, \lambda_{0}\right)=\int_{-\infty}^{\infty} \dot{m}\left(\lambda-\lambda_{0}\right) \exp \left(\frac{-i s^{2}}{4 \pi D}\left(\lambda-\lambda_{0}\right) \operatorname{sgn} s\right) \mathrm{d}\left(\lambda-\lambda_{0}\right) .
$$

For convenience we shall temporarily refer to $R\left(s, \lambda_{0}\right)$ and related functions dropping the $\lambda_{0}$. Now, any function, $f(x)$, can be separated into its even and odd parts defined by

$$
f_{e}(x)=\frac{f(x)+f(-x)}{2}
$$

and

$$
f_{o}(x)=\frac{f(x)-f(-x)}{2}
$$

Let us assume that we have chosen some $\lambda_{0}$ and separated $\dot{m}\left(\lambda-\lambda_{0}\right)$ into its odd and even parts, referred to hereafter as $m_{e}(l)$ and $m_{o}(l)$, respectively. Now

$$
\begin{aligned}
R(s) & =\int_{-\infty}^{\infty} m_{e}(l) \exp \left(\frac{-i s^{2}}{4 \pi D} l \operatorname{sgn} s\right) \mathrm{d} l \\
& +\int_{-\infty}^{\infty} m_{o}(l) \exp \left(\frac{-i s^{2}}{4 \pi D} l \operatorname{sgn} s \mathrm{~d} l\right) \\
& =\int_{-\infty}^{\infty} m_{e}(l) \cos \left(\frac{-s^{2}}{4 \pi D} l \operatorname{sgn} s\right) \mathrm{d} l \\
& -i \int_{-\infty}^{\infty} m_{o}(l) \sin \left(\frac{-s^{2}}{4 \pi D} l \operatorname{sgn} s\right) \mathrm{d} l \\
& =2 \int_{0}^{\infty} m_{e}(l) \cos \left(\frac{-s^{2}}{4 \pi D} l \text { sgn } s\right) \mathrm{d} l \\
& =R_{e}(s)+R_{o}(s), \frac{w h e r e ~ e \text { and } o \text { denote evenness and oddness }}{\text { respectively, as can be seen by changing the sign of } s .} \\
& -2 i \int_{0}^{\infty} m_{o}(l) \sin \left[\frac{-s^{2}}{4 \pi D} l \text { sgn } s\right] \mathrm{d} l
\end{aligned}
$$




$$
\begin{aligned}
r\left(\theta, \lambda_{0}\right) & =\frac{1}{2 \pi} \int_{-\infty}^{\infty} R_{e}(s) e^{i s \theta} \mathrm{d} s \\
& +\frac{1}{2 \pi} \int_{-\infty}^{\infty} R_{o}(s) e^{i s \theta} \mathrm{d} s \\
& =\frac{1}{\pi} \int_{0}^{\infty} R_{e}(s) \cos (s \theta) \mathrm{d} s \\
& +\frac{i}{\pi} \int_{0}^{\infty} R_{o}(s) \sin (s \theta) \mathrm{d} s \\
& =r_{e}\left(\theta, \lambda_{0}\right)+r_{o}\left(\theta, \lambda_{0}\right) .
\end{aligned}
$$

Thus we come to the conclusion that $r\left(\theta, \lambda_{0}\right)$ is purely real and consists of an even and odd part, which is the result of the original even and odd parts of $\dot{m}(l)$ going through the transformations and change of variable from $s^{2}$ to $s$ independently.

This being so, it should be our objective to maximize $r_{e}\left(\theta, \lambda_{0}\right)$ and minimize $r_{o}\left(\theta, \lambda_{0}\right)$, which is done by choosing the centroid of the function as the zero. The wavelength corresponding to the centroid is taken as $\lambda_{0}$ to be used to calculate $-p^{\prime \prime}\left(-\theta, \lambda_{0}\right)$.

The Fourier transforms can be performed numerically and $r(\theta), r_{e}(\theta)$, and $r_{o}(\theta)$ calculated for the $\lambda_{0}$ chosen.

The restored distribution may be divided into its odd and even parts and should represent $t_{m}\left(\theta, \lambda_{0}\right) * r_{e}\left(\theta, \lambda_{0}\right)$ and $t_{m}\left(\theta, \lambda_{0}\right) * r_{o}\left(\theta, \lambda_{0}\right)$, respectively. If, as can be reasonably assumed, the stellar brightness distribution is taken to be circularly symmetrical and lacking in any bright spots, then the even part of the restored distribution yields a one-dimensional measure of the distribution.

In practice, wrong zeros, wrong rates, and wrong temperatures will induce effects on the even and odd parts, which as we shall see can be corrected for iteratively.

\section{An Illustration of the Restoration Process}

I have attempted, in a preliminary approach, to apply the method above to the reduction of the observation of the occultation of $\lambda$-Aquarii, a type M2 III star, reported by Nather et al. (1970). The observed counts with a modified 400 channel multiscalar taken at 2 msec per channel are shown in Figure 3.

Dr Evans informed me that the spectral response of the system could be taken to be that by Johnson (1955) for a V filter in conjunction with a 1P21 photomultiplier tube as shown in Figure 4. He also supplied the predictions:

Predicted rate: $0.7500 \mathrm{~m} / \mathrm{msec}$.

Topocentric distance: $3.67474 \times 10^{8} \mathrm{~m}$. 


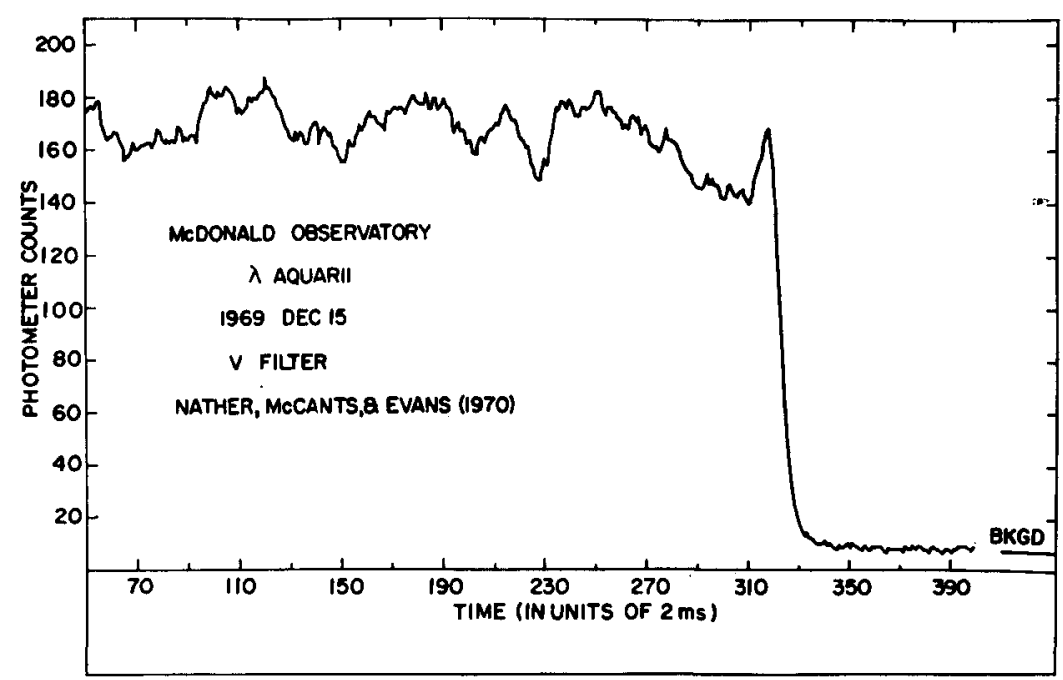

Fig. 3. The occultation curve of $\lambda$-Aquarii.

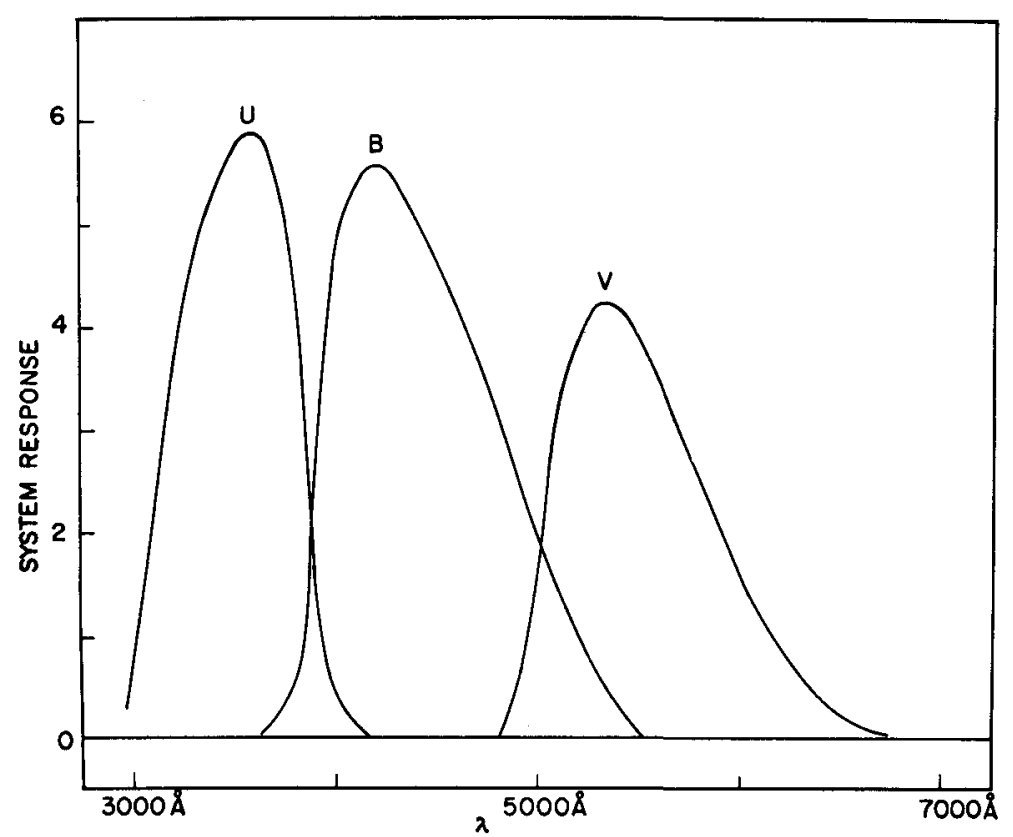

Fig. 4. The system responses for U, B, and V filters used in conjunction with a 1P21 photomultiplier (after Johnson, 1955). The response assumed is that for the $\mathrm{V}$ filter. 
Lunar direction: $53^{\circ} .9$.

Position angle: $21^{\circ} .0$.

To begin with, the temperature was assumed to be $3350 \mathrm{~K}$ and the total normalized spectral response, $\dot{m}(l)$, was computed. The centroid of the response was found to be $5583.59 \AA$. The theoretically correct response for the filter was found in two ways which both agreed;

(a) by going through the Fourier transform and change of variable process from $\dot{m}(l)$ to $r\left(\theta, \lambda_{0}\right)$ centering $\dot{m}(l)$ at the assumed wavelength, and

(b) by summing the response to a unit point source over the spectral response and restoring for $-p^{\prime \prime}\left(-\theta, \lambda_{0}\right)$ at $\lambda_{0}=5583.59 \AA$.

The predictions were assumed to be correct for these calculations which were suitably scaled. The convolution was carried out with the zero at the $25 \%$ point of the assumed response and the result is shown in Figure 5. The odd and even parts were normalized with respect to the maximum for $r\left(\theta, \lambda_{0}\right)$.

The even part has a half-width of $1.8125 \times 10^{-3}$ arcsec and a first negative side-lobe level of the order of $10 \%$. The odd part has a maximum side-lobe level of some $8 \%$.

In order to understand the errors that could arise, the rate of motion of the source was varied from 0.91 to 1.09 times the correct rate. The results are shown in Figure 6. The predominant features are the decrease in the relative amplitude of the even part at zero accompanied by a rapid rise in the values of the odd side-lobes.

The change of centroid, $\lambda_{0}$, with temperature was calculated and is shown in Figure 7. The effects were negligible within a temperature range of $3000 \mathrm{~K}$ to $3600 \mathrm{~K}$ indicating that the stellar spectrum has little effect with this star observed with this particular combination of filter and photoelectric photometer.

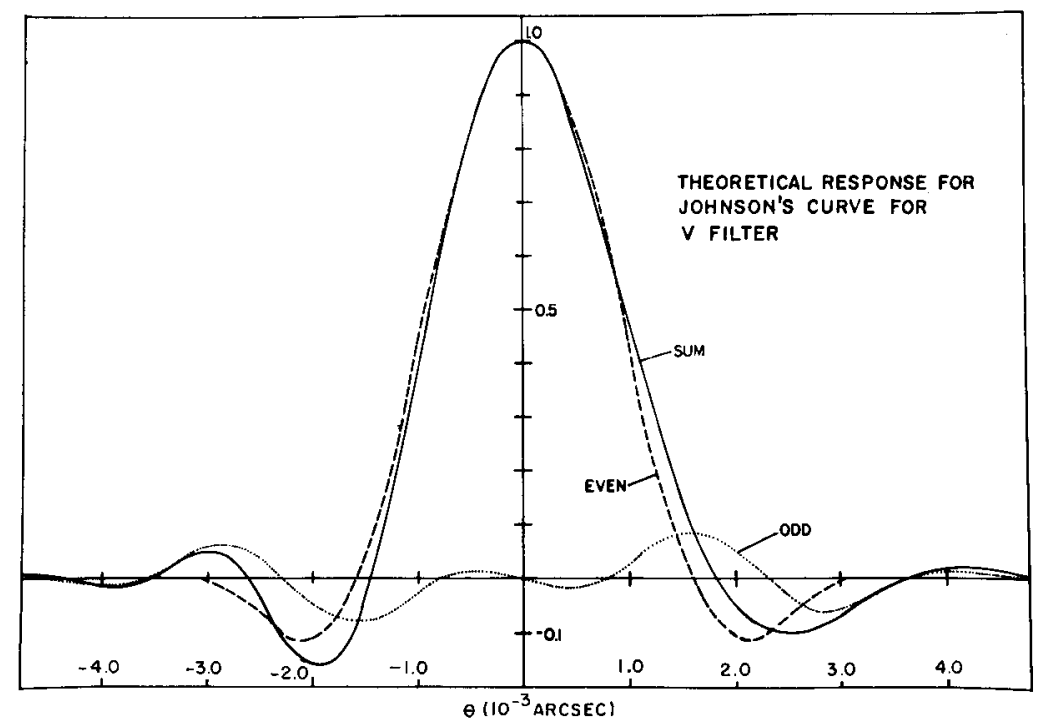

Fig. 5. The calculated response to a unit point source at temperature $T=3350 \mathrm{~K}$ using the $\mathrm{V}$ filter. 

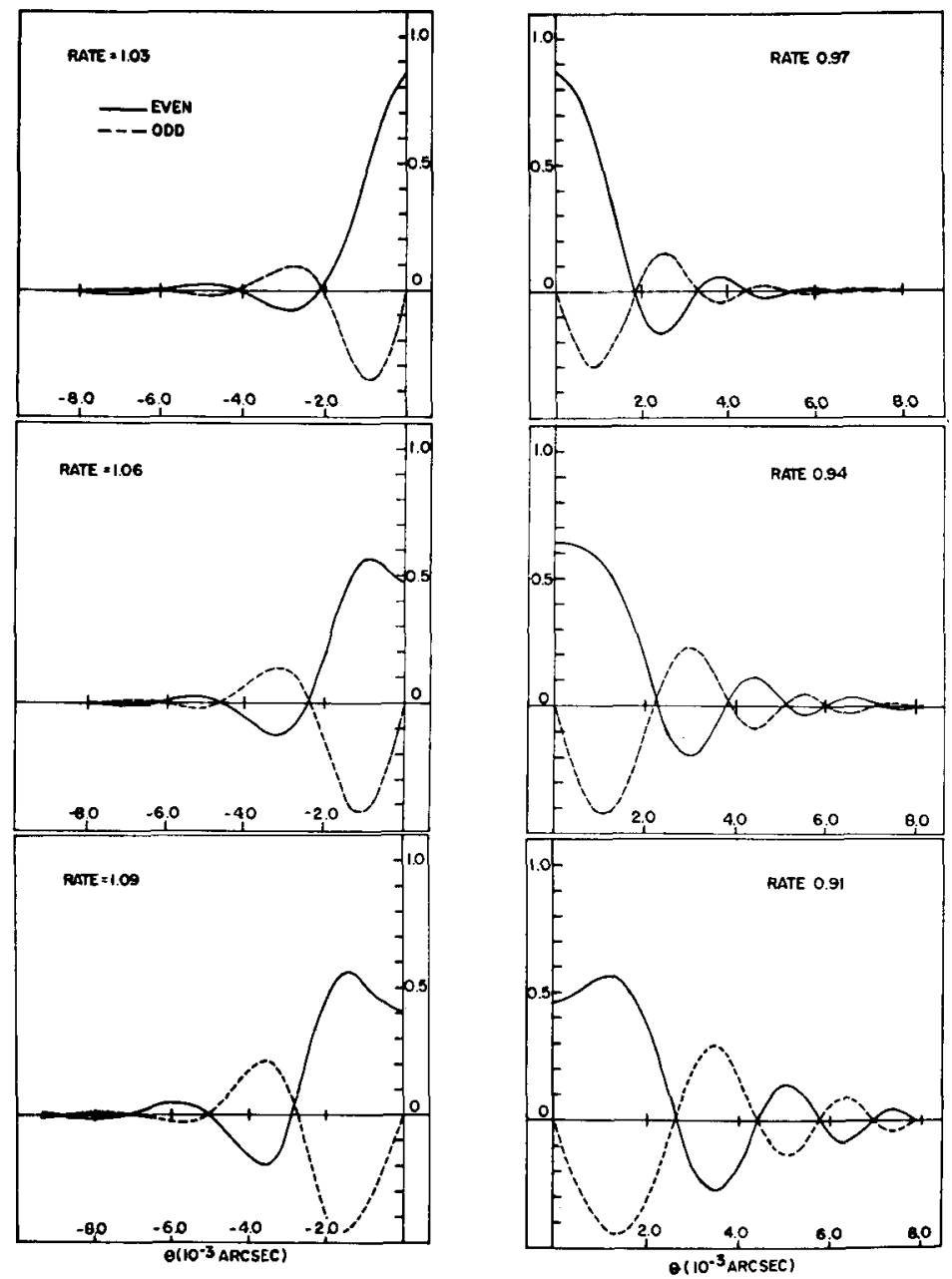

Fig. 6. The calculated odd and even responses to a unit point source at $T=3350 \mathrm{~K}$, using the $\mathrm{V}$ filter, for different rates proportional to the true rate (Note; only half the response is depicted for each rate).

The actual data for $\lambda$-Aquarii were then restored for the assumed temperature of $3350 \mathrm{~K}$, using the predicted parameters and the $25 \%$ point as zero; the result is shown in Figure 8 , in terms of the odd and even parts normalized as before - a convention we use throughout. It can be seen that though the even part has a central value of unity, it is funnily shaped and that the odd part is high. This curve was, however, used to estimate the size of the star, which in turn can be used to modify the zero. Assuming that the variances of the stellar distribution (assumed to be gaussian) and the even part of the theoretical part of the theoretical point source response add, the half width of the gaussian was found to be $6.0 \times 10^{-3}$ arcsec. The diameter of a uniform disk of the same equivalent width as the gaussian was calculated. The one- 


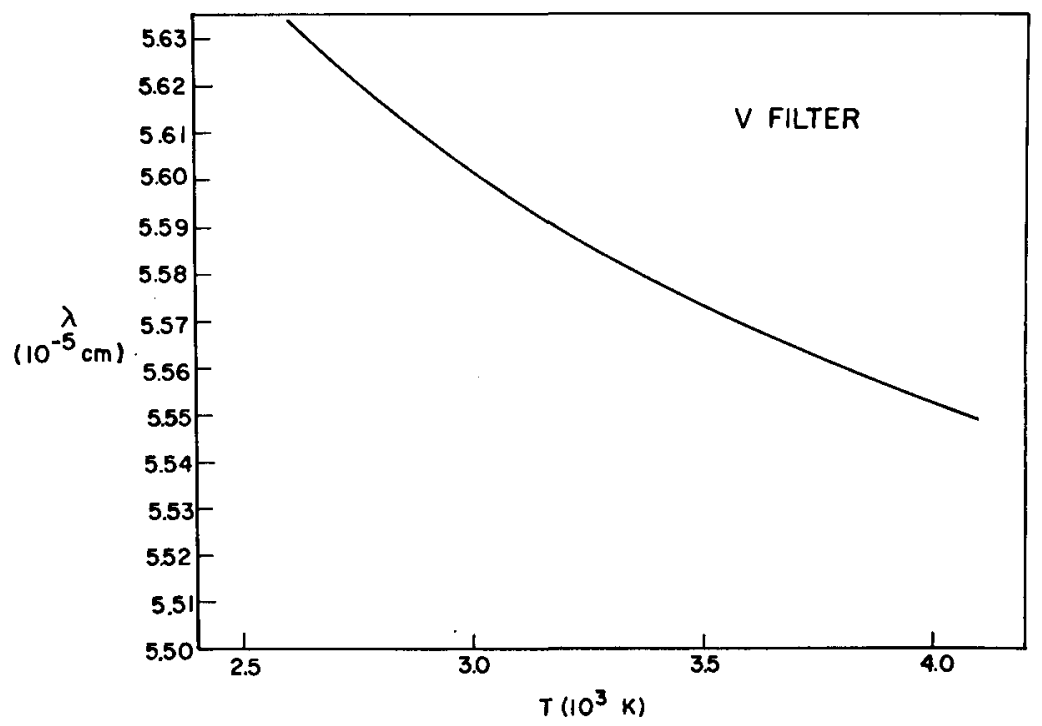

Fig. 7. The centroid wavelength of the total spectral response versus stellar temperature.

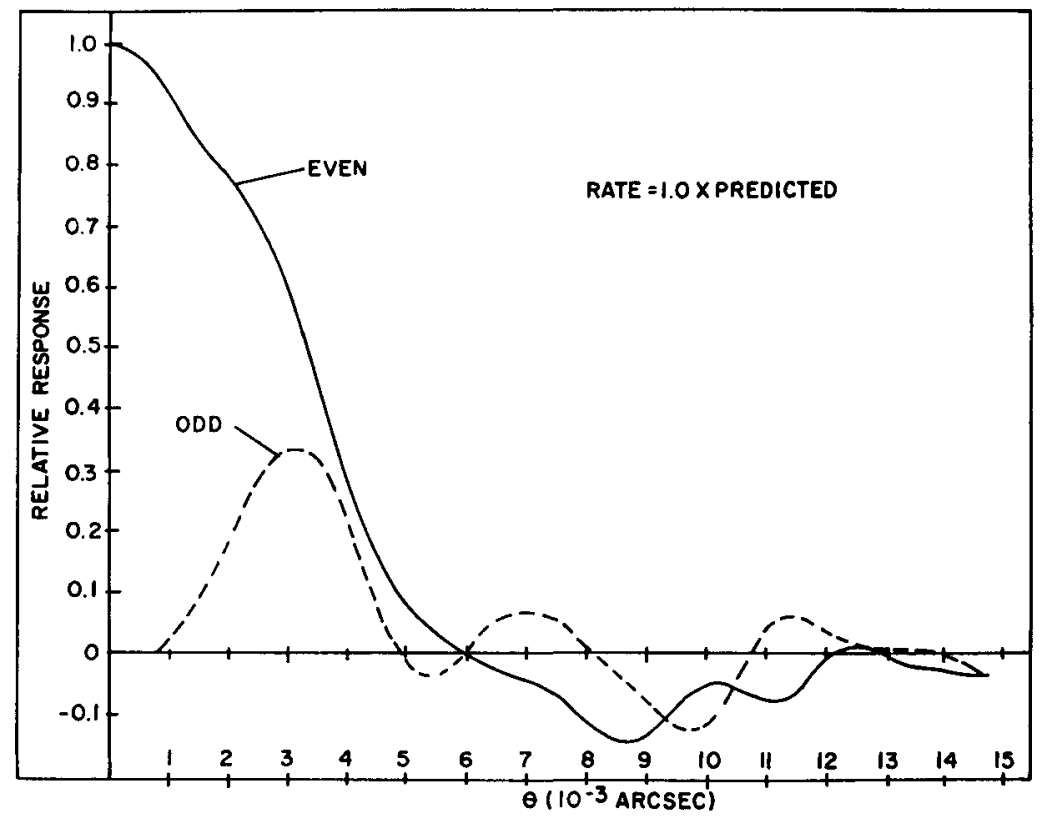

Fig. 8. The odd and even parts of the restored distribution for $\lambda$-Aq assuming the predictions to be correct. 
dimensional disk distribution was convolved with the theoretical summed Fresnel response to find the zero. It was found that this would be located at the $29 \%$ value of the observed curve.

Various restorations, at differing rates and slightly differing zeros were attempted, with the aim being to maximize the central even response and minimize the odd sidelobes. The best response found within the time available is displayed in terms of its odd and even parts in Figure 9, corresponding to a rate 0.94 times the predicted rate.

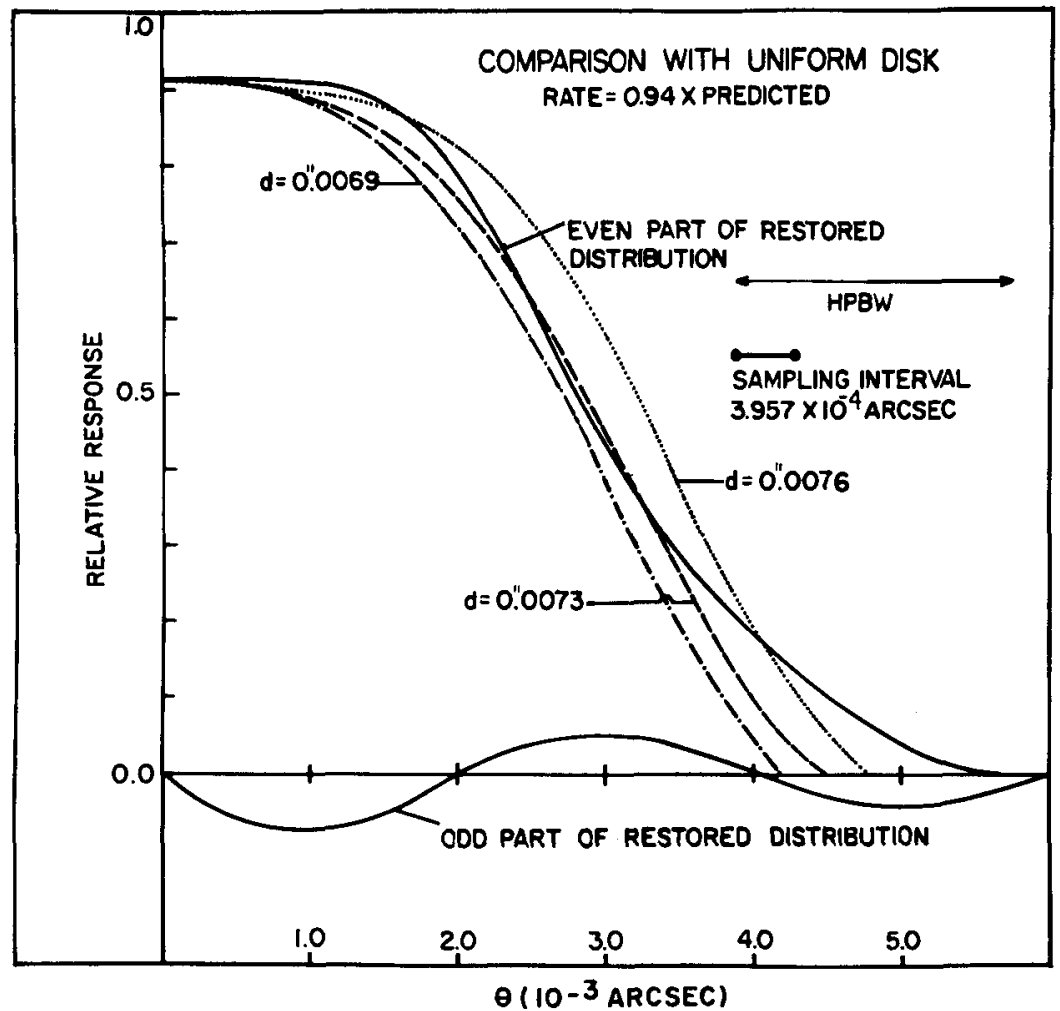

Fig. 9. Comparison of the best restoration (odd and even parts) with calculated uniform disk distributions.

The even part has not the maximal central response expected. Further, the odd part has a maximum value of some $5 \%$ and has a nearly sinusoidal shape with a half wavelength equal to the theoretical resolution of the even part. It should in fact be zero, given the smoothing of the broadish source. I have not yet been able to find out why this should be so - it could arise from spiky irregularities in the data, or from a wrong assumption of the shape of the spectral response of the system.

If we assume, for the moment, that this is the best we can do, the rate leads to a conclusion that lunar limb is inclined to the predicted level limb at an angle com- 
parable to that quoted by Nather et al. (1970) - their rate of motion is 0.9437 times the predicted rate.

In order to obtain an idea of the stellar diameter, I have convolved the strip distributions due to three differently sized uniform circular disks with the theoretical even point source response. It can be seen that the best fit is close to the value of 0.0074 quoted by Nather et al. from model fitting.

There is some indication that there is center-to-limb variation, but it would be advisable to refine the restoration further before any conclusions are drawn.

I noted while trying these model convolutions, that with the narrow theoretical even response predicted in this case a central dip of the even response could occur when the size of the uniform disk is about $0^{\prime \prime} 0080$ - the error in the odd part could arise from attempting to maximize the central part of the even response. The correct procedure is perhaps to minimize the odd part as much as possible, and in the case of narrow sources, compare its level with that theoretically predicted.

The iterative procedure adopted appears to work reasonably. The exact order for each observation should be determined with model trials of restoration as we have done here.

The substantive improvements of the method used over the 'Scheuer-Von Hoerner' method lie in the following features:

(a) direct deconvolution, without smoothing in advance with a well-behaved function, can be undertaken, with calculable effective equivalent beams and

(b) the appropriate wavelength, $\lambda_{0}$, for the restoring function, $-p^{\prime \prime}\left(\theta, \lambda_{0}\right)$, can be chosen for optimal beamwidth and sensitivity, by trial and error.

\section{Acknowledgements}

Part of this work was done while I was a National Academy of Sciences/National Research Council Senior Research Associate with the Radio Astronomy Branch of NASA, Goddard Space Flight Center. My thanks are in particular due to Miss Jacqueline Hard for assistance with the computing, and to Dr Richard Hartle for valuable discussions.

I gratefully acknowledge the assistance of Dr T. J. Deeming, who read this paper at Brighton in my absence.

\section{A Note Added after Presentation of the Paper}

A fundamental limitation to this method of restoration is its assumption of a circularly symmetric stellar brightness distribution, as has been brought to my attention by some comments made by Dr D. S. Evans. This can be understood, as follows, in the terminology of the paper.

In general, the restored distribution represents

$$
t_{m}\left(\theta, \lambda_{0}\right) * r\left(\theta, \lambda_{0}\right)=\left(t_{m_{e}}\left(\theta, \lambda_{0}\right)+t_{m_{o}}\left(\theta, \lambda_{0}\right)\right) *\left(r_{e}\left(\theta, \lambda_{0}\right)+r_{o}\left(\theta, \lambda_{0}\right)\right)
$$


If circularly symmetry is assumed, $t_{m o}\left(\theta, \lambda_{0}\right)=0$, and the even part of the restored distribution represents the stellar distribution convolved with $r_{e}\left(\theta, \lambda_{0}\right)$.

However, if an odd part exists in the stellar distribution it contributes a term equalling $t_{m o}\left(\theta, \lambda_{0}\right) * r_{o}\left(\theta, \lambda_{0}\right)$, (which is inseparable from the contribution of the even part), to the even part of the restored distribution.

In this sense, the method is not superior to that of model-fitting, and it is not easy to see how to overcome this apparently inherent limitation.

The enhanced odd part in the restoration of the occultation of $\lambda$-Aquarii, given its large width, could very well be an indication of the existence of an asymmetrical component in the stellar distribution.

\section{References}

Berg, R. A.: 1960, 'Stellar Angular Diameters from Lunar Occultations', Ph.D. dissertation, Leander McCormick Observatory, University of Virginia.

Cohen, M. H.: 1969, Ann. Rev. Astron. Astrophys. 6 (Palo Alto, Calif.: Ann. Rev.), pp. 13-38.

Cousins, A. W. J. and Guelke, R.: 1953, Monthly Notices Roy. Astron. Soc. 113, 776.

Eddington, A. S.: 1909, Monthly Notices Roy. Astron. Soc. 69, 178.

Evans, D. S.: 1951, Monthly Notices Roy. Astron. Soc. 111, 64.

Evans, D. S.: 1955, Monthly Notices Roy Astron. Soc. 115, 466.

Evans, D. S.: 1957, Astron. J. 62, 83.

Evans, D. S., Heydenrych, J. C. R., and Van Wyck, D. N.: 1953, Monthly Notices Roy. Astron. Soc. $113,781$.

Hoerner, S., Von.: 1964, Astrophys. J. 140, 65.

Johnson, H. L.: 1955, Ann. Astrophys. 18, 4, 292.

Krishnan, T.: 1970, J. Inst. Tellcom. Engrs. (India) 16, 61 and NASA Technical Note, NASA TN D-5679.

Lang, K. R.: 1969, Astrophys. J. 158, 1189.

McNahon, P. A.: 1908, Monthly Notices Roy. Astron. Soc. 69, 126.

Nather, R. E., McCants, M. M., and Evans, D. S.: 1970, Astrophys. J. (Letters) 160, L181.

Scheuer, P. A. G.: 1962, Australian J. Phys. 15, 333.

Scheuer, P. A. G.: 1965, Monthly Notices Roy. Astron. Soc. 129, 1965.

Sutton, J.: 1966, Ph.D. Thesis, University of Sydney.

Taylor, J. H.: 1966, Nature 210, 1105.

Whitford, A. E.: 1939, Astrophys. J. 89, 472.

Williams, J. D.: 1939, Astrophys. J. 89, 467. 